\title{
Editorial
}

\section{Screening tests before surgery in children}

Safety for the anaesthetised patient is our prime consideration today, and indeed "Safety" has been chosen as a main topic for the Tenth World Congress of Anaesthesiology to be held in 1992. A recent trend has been to question the established routine procedures of our speciality. One by one these are being re-examined to determine whether they are of real value and add to the safety of anaesthesia or are performed simply as a ritual. The necessity for preoperative blood and urine testing has come under such scrutiny and on page 700 of this issue the matter of the routine preoperative haemoglobin estimation in paediatric patients is discussed. This paper raises important questions: is it useful to test every apparently healthy child for the presence of anaemia? If so, is this because mild asymptomatic anaemia increases the risk of a wellconducted general anaesthetic or does indeed require any special considerations?

Several studies have examined the incidence of anaemia (usually defined as $\mathrm{Hb}<100 \mathrm{~g} \cdot \mathrm{L}^{-1}$ ) in paediatric surgical populations. ${ }^{1-4}$ The reported incidence was commonly about $0.5 \%$, but was as high as $1.9 \%$ in one study from a hospital which may service patients from a lower socio-economic level. ${ }^{3}$ Reports also indicate that age (infants under one year), sex (adolescent females), and the presence of chronic diseases (e.g., rheumatoid arthritis) may influence the likelihood of anaemia. All studies appear to indicate that significant anaemia is very rare in otherwise totally healthy, well nourished, active surgical patients. Why, then, subject all these children to a painful and upsetting finger-prick or venepuncture? Can we reliably detect anaemia in children by means of the medical history and physical examination alone? A recent study indicated that mild degrees of anaemia will likely not be detected if the blood test results are not available. ${ }^{2}$ Severe anaemia may be less likely to be missed if a detailed history is taken and a careful physical examination performed. Therefore, if it is indeed necessary to document mild degrees of preoperative anaemia, a blood test is essential. But is it necessary to document mild degrees of anaemia?

Children's Hospital of Los Angeles, University of Southern California.
Does mild anaemia affect the risk of general anaethesia and need we modify our techniques in any way to accommodate the mildly anaemic state? Reported studies all indicate that mild anaemia is not usually considered a reason to postpone minor paediatric surgery. General anaesthesia in these patients is described as uneventful with no suggestion that the usual anaesthetic techniques were modified in any way. A well-conducted general anaesthetic should not threaten, at any time, to reduce oxygen delivery to the tissues by a larger measure than the anaesthesia-induced reduction in the tissue metabolic rate for oxygen. A recently published consensus conference on perioperative red blood cell transfusion concluded that mild anaemia was not associated with perioperative morbidity. ${ }^{5}$ Does it contribute to the general health care of the child to perform a screening test for anaemia when he or she attends the hospital for a minor surgical procedure? Do we find other blood diseases which may require further investigation and therapy? The suggestion from one study is that there is poor follow-up of children found to be mildly anaemic on routine preoperative screening, ${ }^{3}$ hence it is dubious whether routine blood testing contributes much to the overall care of the child.

What is the usual practice in the children's hospitals in Canada at present? Responses from departments in 14 children's hospitals or university paediatric centres across eight provinces indicate that ten of those responding continue to perform routine preoperative haemoglobin or haematocrit determinations on every child. However, eight of these ten departments are located in provinces where legislation requires such preoperative testing (Ontario, Quebec, Saskatchewan). It is interesting that the wisdom of this legislation has not been questioned at all until quite recently. In the United States neither the Joint Committee on Hospital Accreditation nor the American Society of Anesthesiologists suggest that there should be universal requirements for routine preoperative laboratory testing of any patients. Four Canadian paediatric anaesthesia departments do not perform a routine preoperative blood test on every child, but they all require such testing for specific indications (young infants, chronic disease, surgery which may incur blood loss, etc.). Only one of the 14 departments would postpone surgery in an 
otherwise healthy child if the haemoglobin level was less than $100 \mathrm{~g} \cdot \mathrm{L}^{-1}$. Other departments would postpone surgery only if more severe anaemia was detected (haemoglobin level $70-90 \mathrm{~g} \cdot \mathrm{L}^{-1}$ ).

What then should we do? We know that many children are upset by having a blood test. The evidence is that in the case of otherwise healthy children the results of this test are not going to affect our management of their anaesthetic or contribute to their health care in any way. There is a growing movement to omit all blood tests in such children. Provincial legislation which demands unnecessary routine preoperative testing should be revised. Groups of patients who are at risk for more severe and physiologically important anaemia should be tested. These would include all infants, patients with any chronic disease, and those demonstrating any symptoms or signs suggestive of anaemia. Children whose surgery may result in any considerable blood loss should have preoperative testing to establish a baseline. Patients at risk of having a haemoglobinopathy (e.g., Sickle Cell disease) should also be screened. In addition, we should assess very carefully all our patients before surgery to ensure that those who may have any symptoms or signs of disease are identified. Routine testing: no! A careful preoperative assessment considering all aspects of the health of the child and of the intended surgery followed by the appropriate requisition of any indicated tests: yes!

\section{Tests de routine en anesthésie pédiatrique}

En anesthésie, la sécurité des patients nous tient à cœur et d'ailleurs elle constituera le thème $\mathrm{du}$ dixième congrès mondial des anesthésistes qui se tiendra en 1992. Depuis quelques temps, on tend à révvaluer la pertinence des examens de routine pré-opératoire, à faire la part de ceux qui ajoutent à la sûreté de l'anesthésie et de ceux dont l'usage tient du rituel. Il en va ainsi des tests d'urine et de sang et plus particulièrement de la mesure de l'hémoglobine avant une intervention chirurgicale chez l'enfant (voir l'article en p. 700). Les auteurs de l'article en question se demandent s'il est utile de tester tous les enfants apparemment en bonne santé à la recherche d'une anémie asymptomatique el en quoi cette dernière augmenterait le risque anesthésique ou requerrait des précautions particulières.

On évalue l'incidence de l'anémie chez les enfants ( $\mathrm{Hb}$ $\left.<100 \mathrm{~g} \cdot \mathrm{L}^{-1}\right)$ à près de $0,5 \% \%^{1,2,4}$ mais elle peut aller jusqu' à $1,9 \%$ dans les milieux défavorisés. ${ }^{3}$ Elle est aussi plus élevée chez les enfants de moins d'un an, chez les adolescentes et chez les victimes de maladie chronique (arthrite rhumatoïde). Toutes les études s'entendent sur l'extrême rareté d'une anémie importante chez les candidats à une chirurgie qui sont bien nourris, actifs et en bonne santé. Pourquoi donc alors leur infliger une douloureuse piqûre? L'anamnèse et l'examen physique ne suffisent-ils pas à détecter l'anémie chez les enfants? De fait, une étude récente suggère qu'on peut passer à coté d'une anémie légère si on ne mesure pas l'hémoglobine. ${ }^{2}$ Toutefois cela est peu probable dans le cas d'une anémie importante. Est-il important alors avant une intervention chirurgicale d'identifier les cas d'anémie légère?

Ce degré d' anémie augmente-t'il le risque associé à une anesthésie générale ou exige-t'il que l'on modifie nos techniques? Dans aucun des articles de la bibliographie considère t'on que ce soit une raison de retarder l'intervention. De plus, on décrit comme facile le déroulement normal d'une anesthésie générale dans ce contexte. Une anesthésie générale bien faite ne devrait d'ailleurs en aucun temps diminuer la capacité de transport de l'oxygène au niveau tissulaire au delà de la diminution de la demande métabolique en oxygène. D'ailleurs, dans une conférence récente sur les transfusions, on a reconnu que l'anémie légère n'augmente pas la morbidité périopératoire. ${ }^{5}$ Peut on saisir l'occasion qu'offre une intervention mineure pour dépister une anémie légère ou une maladie du sang que l'on traitera? Au moins une étude ${ }^{3}$ indique que le suivi des anémies légères découvertes lors de test préopératoires est inégal et que l'impact de ces tests de routine sur le bien-être de l'enfant est négligeable.

Voyons donc quelle est la pratique actuelle dans les hôpitaux canadiens. Dix parmi les quatorze centres pédiatriques universitaires (situés dans huit provinces) qui ont répondu à l'enquête, continuent à faire de routine une mesure de l'hématocrite et de l'hémoglobine chez tous les enfants. Toutefois, huit d'entre eux sont situés dans des provinces ou cela est obligatoire (Ontario, Québec et Saskatchewan). Il est temps de remettre en question la sagesse de ces dispositions législatives. Notons qu'aux Etats-Unis, ni le Comité conjoint d'accréditation hospitalière ni la Société américaine des anesthésistes ne suggèrent d'examen de routine pour quelque patient que ce soit. Quatre départements universitaires d'anesthésie canadien n'exigent que les examens de laboratoires indiqués par une condition particulière (très jeune âge, maladie chronique, chirurgie sanglante, etc.). Les membres d'un seul département sur quatorze retarderaient l'intervention chirurgicale si la concentration de l'hémoglobine était inférieure à $100 \mathrm{~g} \cdot \mathrm{L}^{-1}$ chez un enfant autrement en bonne santé. D'autres fixent la barre un peu plus bas, de 70 à $90 \mathrm{~g} \cdot \mathrm{L}^{-1}$. 
Quelle conduite devrions-nous adopter? Comme on sait que la plupart des enfants n'aiment pas les piqûres et que de toute façon le résultat du test ne changera pas notre conduite envers un enfant en bonne santé, nous devrions abandonner les tests de routine en anesthésie pédiatrique et encourager les législateurs à mettre fin à l'obligation de tester. On devrait cependant continuer à rechercher l'anémie importante chez les groupes à risque tels les nouveaux-nés, les enfants atteints de maladie chronique ou affligés de signes ou de symptômes d'anémie. On devrait aussi établir une valeur de contrôle de l'hémoglobine chez les enfants susceptibles de saigner beaucoup lors de l'opération sans négliger les patients à risque d'hémoglobinopathie (anémie falciforme). Cela nous obligera à porter plus d'attention à l'anamnèse et à l'examen physique en préopératoire, nous devrons être à l'affût des signes et symptômes de l'anémie. Sus aux tests de routine! Saisissons la chance de pouvoir exercer notre jugement au cas par cas.

\section{References}

I Roy WL, Lerman J, McIntyre BG. Preoperative haemoglobin values in minor paediatric surgery. Can J Anaesth 1990; 37: S7.

2 Hackman T, Steward DJ. What is the value of preoperative haemoglobin determinations in paediatric outpatients? Anesthesiology 1989; 71: 1168A.

$3 O^{\prime}$ Connor ME, Drasner $K$. Preoperative laboratory lesting of children undergoing elective surgery. Aneth Analg. 1990; 70: 176-80.

4 Rossello PJ, Cruz AR, Mayol PM. Routine laboratory tests for elective surgery in paediatric patients: are they necessary? Bol Asoc Med P R 1980; 72: 614-23.

5 Consensus Conference. Perioperative Red Blood Cell Transfusion. JAMA 1988; 260: 2700-3. 